\title{
ON TWO FUNDAMENTAL APPROACHES FOR RELIABILITY \\ IMPROVEMENT AND RISK REDUCTION BY USING ALGEBRAIC INEQUALITIES
}

\author{
Michael T. Todinov \\ Oxford Brookes University, Oxford, UK \\ School of Engineering, Computing and Mathematics \\ mtodinov@brookes.ac.uk
}

The paper introduces two fundamental approaches for reliability improvement and risk reduction by using non-trivial algebraic inequalities: (i) by proving an inequality derived or conjectured from a real system or process and (ii) by creating meaningful interpretation of an existing non-trivial abstract inequality relevant to a real system or process. $A$ formidable advantage of the non-trivial algebraic inequalities can be found in the capacity to produce tight bounds related to reliability-critical design parameters in the absence of any knowledge about the variation of the controlling variables. The effectiveness of the first approach has been demonstrated by examples related to decision-making under deep uncertainty and examples related to ranking systems built on components whose reliabilities are unknown.

To demonstrate the second approach, meaningful interpretation has been created for an inequality which is a special case of the Cauchy-Schwarz inequality. By varying the interpretation of the variables, the same inequality holds for elastic elements, resistors and capacitors arranged in series and parallel.

The paper also shows that meaningful interpretation of super-additive and sub-additive inequalities can be used with success for optimising various systems and processes. Meaningful interpretation of super-additive and sub-additive inequalities has been used for maximising the stored elastic strain energy at a specified total displacement and for optimising the profit from an investment.

Finally, meaningful interpretation of an algebraic inequality has been used for reducing uncertainty and the risk of incorrect prediction about the magnitude ranking of sequential random events.

Keywords: risk reduction; reliability improvement; domain-independent methods; algebraic inequalities

\section{INTRODUCTION}

While reliability and risk assessment are truly domain-independent areas, this cannot be stated about the equally important areas of reliability improvement and risk reduction. For decades, the reliability and risk science failed to appreciate that reliability improvement and risk reduction is underpinned by general principles that work in many unrelated domains. Until now, the approach to reliability improvement and risk reduction almost solely relies on knowledge from a specific domain and is conducted exclusively by experts in that specific domain. This is the reason for the strong perception that effective risk reduction can be delivered solely by using methods offered by the specific domain, without resorting to a general risk reduction methodology. As a result, in standard textbooks on mechanical engineering and design of machine components, for example ${ }^{1-7}$, there is no mention of generic (domain-independent) methods for reliability improvement and risk reduction. 
This misconception resulted in ineffective reliability improvement and risk reduction across the entire industry. Valuable opportunities for improving reliability and reducing risk have been overlooked which led to numerous accidents resulting in financial losses, fatalities and damage to the environment.

Recently, a number of domain-independent methods and principles for improving reliability and reducing risk have been formulated ${ }^{8}$. The present paper extends this work by introducing a new domain-independent method for improving reliability and reducing risk based on algebraic inequalities.

There are a number of useful non-trivial algebraic inequalities such as the Arithmetic mean - Geometric mean (AM-GM) inequality, Cauchy-Schwartz inequality, the rearrangement inequality, the Chebyshev's inequality, Jensen's inequality, Muirhead's inequality, etc. Algebraic inequalities have been discussed extensively ${ }^{9-14}$. In probability theory, well-known non-trivial inequalities are the Chebyshev's inequality, Markov's inequality, Boole's inequality, Bonferroni inequalities and Jensen's inequality.

In reliability and risk research, inequalities have been used exclusively as a mathematical tool for reliability and risk evaluation ${ }^{15-22}$ and for characterisation of reliability functions. It is important to guarantee that the reliability of a system meets certain minimal expectations and inequalities have been used ${ }^{15}$ for obtaining lower and upper bounds on the system reliability by using minimal cut sets and minimal path sets. Xie and Lai ${ }^{16}$ used simple conditional inequalities to obtain more accurate approximations for system reliability, instead of the usual minimal cut and minimal cut bounds. By using improved Bonferroni inequalities, lower and upper bound of system reliability have been derived by Makri and Psillakis ${ }^{17}$. Inequalitybased reliability estimates for complex systems have also been proposed by Hill et al ${ }^{18}$.

In the reliability and risk research, simple inequalities have also been used to express relationships between random variables and their transformations to generate insight into the structure of reliability distributions. For example, well-known inequalities about a random variable $X$ with unknown probability distribution are the Chebyshev's inequality and Markov's inequality. Simple inequalities with relation to reliability prediction have been used by Berg and Kesten ${ }^{20}$; inequalities involving expectations have been used by Kundu and Ghosh $^{21}$ for characterising some well-known reliability distributions. In standard reliability textbooks ${ }^{23-26}$ there is a clear lack of discussion related to improving reliability and reducing uncertainty and risk by using non-trivial algebraic inequalities.

Applications of inequalities have also been considered in physics ${ }^{27}$ and engineering ${ }^{28,31}$. However, in the mechanical engineering design literature ${ }^{1-7,28-31}$, there is also a lack of discussion on the use of complex algebraic inequalities to reduce risk. In engineering design, the application of inequalities is mainly confined to design variables required to satisfy various trivial design inequalities in order to guarantee that the design will perform its required functions ${ }^{31}$.

A formidable advantage of non-trivial algebraic inequalities is that they do not normally require knowledge related to the values of the controlling variables entering the inequalities, which makes them ideal for handling deep uncertainty associated with systems, processes and decisions. Non-trivial algebraic inequalities possess the capacity to produce tight bounds related to reliability-critical design parameters (e.g. material properties, dimensions, loads) in the absence of any knowledge of the variation of the controlling variables. This advantage of algebraic inequalities is demonstrated later, where non-trivial algebraic inequalities will be used for ranking system topologies and component configurations in the case where the reliabilities of the components are unknown.

To the best of our knowledge of existing approaches involving inequalities in reliability and risk, non-trivial inequalities have not yet been used as a domain-independent reliability improvement or risk reduction method. 
Another formidable advantage of algebraic inequalities is the possibility to create a meaningful interpretation of an existing abstract inequality in terms of reliability and risk and apply this interpretation to a real system or process. To the best of our knowledge, creating meaningful interpretation for existing non-trivial abstract inequalities and attaching it to a real system or process has not yet been explored in the reliability and risk literature. Covering these gaps constitutes the contribution of this paper.

This paper introduces two fundamental approaches of using non-trivial algebraic inequalities to improve reliability and reduce risk: (i) proving a non-trivial inequality derived or conjectured from analysing a physical system or process and (ii) creating relevant meaning and meaningful interpretation for an existing non-trivial abstract inequality and attaching it to a real system or process.

To demonstrate the first approach, the paper focuses on the application of algebraic inequalities for ranking systems and decision-making alternatives. To demonstrate the second approach, the paper focuses on the application of algebraic inequalities for producing tight bounds for the variation of properties of assemblies in the absence of any knowledge about the properties of the individual parts. An interpretation of an existing abstract inequality has also been provided for maximising the probability of selecting high-reliability components.

Here is a list of techniques that can be used for proving a conjectured algebraic inequality: (i) by algebraic manipulation (ii) by reducing to known inequalities, (iii) using mathematical induction, (iv) by a direct analysis, (v) by proving a simpler intermediate inequality, (vi) by segmentation, (vii) using the properties of convex (concave) functions, (viii) using the rearrangement inequality, (ix) using the method of sub-additive and super-additive functions, (x) using the properties of the quadratic trinomial, (xi) using substitution, (xii) using symmetry (xiii) using homogeneity, (xiv) using methods from calculus, (xv) using the properties of partial derivatives that do not change sign.

\section{USING NON-TRIVIAL INEQUALITIES FOR MAXIMISING THE PROBABILITY OF HIGH-RELIABILITY COMPONENTS}

The first fundamental approach of using inequalities to improve reliability and reduce risk starts with the real system or a process and moves towards deriving/conjecturing an algebraic inequality testing and proving it by using some of the techniques listed in the introduction.

It includes four basic steps (Figure 1): (i) theoretical analysis of the system/process (e.g. by using reliability/risk theory), (ii) deriving or conjecturing an inequality about the competing alternatives or an inequality related to the bounds of a risk-critical parameter, and (iii) testing and proving the conjectured inequality.

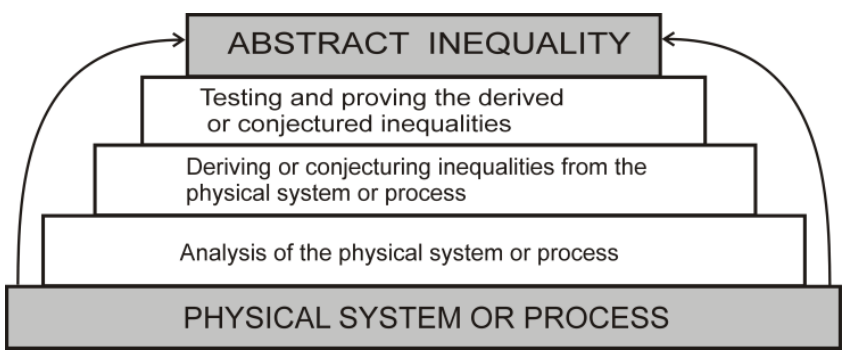

Figure 1. Using non-trivial algebraic inequalities to improve reliability and reduce risk by starting from the physical system and moving towards proving an abstract inequality.

Consider a real-world example featuring a market where three suppliers $A_{1}, A_{2}$ and $A_{3}$, produce high-reliability components of the same type, with probabilities $x_{1}, x_{2}$ and $x_{3}$, 
which are unknown. If two components are to be purchased and installed in a device, the question of interest is which strategy maximises the probability that both components will be highly reliable: (i) taking the two components from the same, randomly selected supplier or (ii) taking the two components from two different randomly selected suppliers. At first glance, it seems that either of these strategies could be chosen because the proportions $x_{1}, x_{2}$ and $x_{3}$ of high-reliability components characterising the suppliers are unknown. Surprisingly, this common-sense conclusion is incorrect.

The probability of purchasing two high-reliability components from the same, randomly selected supplier, is:

$$
p_{1}=(1 / 3) x_{1}^{2}+(1 / 3) x_{2}^{2}+(1 / 3) x_{3}^{2}
$$

This is composed of the probabilities of three mutually exclusive events: (i) the probability (1/3) $x_{1}^{2}$ that supplier $A_{1}$ will be selected and both components taken from $A_{1}$ will be highly reliable; (ii) the probability $(1 / 3) x_{2}^{2}$ that supplier $A_{2}$ will be selected and both components taken from $A_{2}$ will be highly reliable and (iii) the probability $(1 / 3) x_{3}^{2}$ that supplier $A_{3}$ will be selected and both components taken from $A_{3}$ will be highly reliable.

Accordingly, the probability of purchasing two high-reliability components from two randomly selected suppliers is:

$$
p_{2}=(1 / 3) x_{1} x_{2}+(1 / 3) x_{1} x_{3}+(1 / 3) x_{2} x_{3}
$$

This is composed of the probabilities of three mutually exclusive events: (i) the probability (1/3) $x_{1} x_{2}$ that suppliers $A_{1}$ and $A_{2}$ will be selected and both components taken from $A_{1}$ and $A_{2}$ will be highly reliable; (ii) the probability (1/3) $x_{1} x_{3}$ that suppliers $A_{1}$ and $A_{3}$ will be selected and both components taken from $A_{1}$ and $A_{3}$ will be highly reliable and (iii) the probability (1/3) $x_{2} x_{3}$ that suppliers $A_{2}$ and $A_{3}$ will be selected and both components taken from $A_{2}$ and $A_{3}$ will be highly reliable.

The question of interest can be answered by comparing the probabilities $p_{1}$ and $p_{2}$ which is equivalent to proving the conjectured non-trivial algebraic inequality

$$
(1 / 3) x_{1}^{2}+(1 / 3) x_{2}^{2}+(1 / 3) x_{3}^{2} \geq(1 / 3) x_{1} x_{2}+(1 / 3) x_{2} x_{3}+(1 / 3) x_{3} x_{1}
$$

This inequality can be proved rigorously by using a number of different techniques: by a direct manipulation, by using segmentation, by using the rearrangement inequality or by reducing it to a standard inequality (e.g. to the Muirhead's inequality).

The segmentation technique is a powerful technique whose essence is to segment (split) the original inequality into simple parts and express each part by using some of the standard inequalities. Adding the separate parts assembles the original inequality and completes the proof. Proving inequality (1) is equivalent to proving the inequality

$$
x_{1}^{2}+x_{2}^{2}+x_{3}^{2} \geq x_{1} x_{2}+x_{2} x_{3}+x_{3} x_{1}
$$

because inequality (1) can be obtained from inequality (2) by dividing both sides of (2) to the positive constant ' 3 '. Inequality (2) can be segmented into three parts by using the standard arithmetic mean - geometric mean (AM-GM) inequality:

$$
\frac{x_{1}^{2}+x_{2}^{2}}{2} \geq \sqrt{x_{1}^{2} x_{2}^{2}}=x_{1} x_{2}, \frac{x_{2}^{2}+x_{3}^{2}}{2} \geq \sqrt{x_{2}^{2} x_{3}^{2}}=x_{2} x_{3} \text { and } \frac{x_{3}^{2}+x_{1}^{2}}{2} \geq \sqrt{x_{3}^{2} x_{1}^{2}}=x_{3} x_{1} \text {. }
$$

Adding the three inequalities gives the original inequality (2) and completes the proof of inequality (1). 
This is a surprising and highly counter-intuitive result. After all, the proportions of highreliability components characterising the suppliers are unknown. Despite the total lack of knowledge related to the proportions of high-reliability components characterising the separate suppliers and regarding existing dependencies related to the proportions of highreliability components characterising the suppliers, inequality (1) still holds. The reduced epistemic uncertainty allows an appropriate choice to be made, associated with reduced risk of not selecting two high-reliability components.

The same approach can be generalised for a larger number of purchased components. If, for example, three components are to be purchased from three suppliers $(n=3)$ and installed in an assembly, the question of interest is to choose between several competing strategies: a) purchasing the three components from a single, randomly selected supplier; $b$ ) purchasing the three components from all three available suppliers; or c) purchasing the three components from two randomly selected suppliers. Suppose that the suppliers are characterised by proportions $x_{1}, x_{2}$ and $x_{3}$ of high-reliability components and unknown interdependences of the proportions of high-reliability components among the suppliers.

Proving that strategy ' $a$ ' is better than strategy ' $b$ ' reduces to proving the inequality

$$
\frac{1}{3} x_{1}^{3}+\frac{1}{3} x_{2}^{3}+\frac{1}{3} x_{3}^{3} \geq x_{1} x_{2} x_{3}
$$

The left hand side of inequality (3) is the probability of purchasing three high-reliability components from a randomly selected supplier. The right side of inequality (3) is the probability of purchasing three high-reliability components from the three available suppliers. Inequality (3) can be proved by a direct application of the AM-GM inequality:

$$
\frac{x_{1}^{3}+x_{2}^{3}+x_{3}^{3}}{3} \geq \sqrt[3]{x_{1}^{3} x_{2}^{3} x_{3}^{3}}=x_{1} x_{2} x_{3}
$$

Proving that strategy ' $a$ ' is better than strategy ' $c$ ' reduces to proving the inequality

$$
\begin{aligned}
& (1 / 3) x_{1}^{3}+(1 / 3) x_{2}^{3}+(1 / 3) x_{3}^{3} \geq(1 / 6) \times x_{1}^{2} x_{2}+ \\
& (1 / 6) \times x_{1}^{2} x_{3}+(1 / 6) x_{2}^{2} x_{1}+(1 / 6) \times x_{2}^{2} x_{3}+(1 / 6) x_{3}^{2} x_{1}+(1 / 6) x_{3}^{2} x_{2}
\end{aligned}
$$

The left hand side of inequality (4) is the probability of purchasing three high-reliability components from a randomly selected single supplier. The right hand side of inequality (4) is the probability of purchasing three high-reliability components from two randomly selected suppliers.

Applying the AM-GM inequality six times, yields the following relationships:

$$
\begin{aligned}
& \frac{x_{1}^{3}+x_{1}^{3}+x_{2}^{3}}{3} \geq \sqrt[3]{x_{1}^{3} x_{1}^{3} x_{2}^{3}}=x_{1}^{2} x_{2}, \frac{x_{2}^{3}+x_{2}^{3}+x_{1}^{3}}{3} \geq \sqrt[3]{x_{2}^{3} x_{2}^{3} x_{1}^{3}}=x_{2}^{2} x_{1}, \\
& \frac{x_{2}^{3}+x_{2}^{3}+x_{3}^{3}}{3} \geq \sqrt[3]{x_{2}^{3} x_{2}^{3} x_{3}^{3}}=x_{2}^{2} x_{3}, \frac{x_{3}^{3}+x_{3}^{3}+x_{2}^{3}}{3} \geq \sqrt[3]{x_{3}^{3} x_{3}^{3} x_{2}^{3}}=x_{3}^{2} x_{2} \\
& \frac{x_{3}^{3}+x_{3}^{3}+x_{1}^{3}}{3} \geq \sqrt[3]{x_{3}^{3} x_{3}^{3} x_{1}^{3}}=x_{3}^{2} x_{1}, \frac{x_{1}^{3}+x_{1}^{3}+x_{3}^{3}}{3} \geq \sqrt[3]{x_{1}^{3} x_{1}^{3} x_{3}^{3}}=x_{1}^{2} x_{3}
\end{aligned}
$$

Adding the six inequalities and dividing both sides with the positive constant 6 gives inequality (4) and completes the proof.

These results are confirmed by an alternative approach based on the Muirhead's inequality. Muirhead's inequality: If the sequence $\{a\}$ majorizes the sequence $\{b\}$ and $x_{1}, x_{2}, \ldots, x_{n}$ are non-negative, the inequality

holds ${ }^{11}$.

$$
\sum_{\text {sym }} x_{1}^{a_{1}} x_{2}^{a_{2}} \ldots x_{n}^{a_{n}} \geq \sum_{\text {sym }} x_{1}^{b_{1}} x_{2}^{b_{2}} \ldots x_{n}^{b_{n}}
$$


Consider the two non-increasing sequences $a_{1} \geq a_{2}, \ldots, \ldots a_{n}$ and $b_{1} \geq b_{2}, \ldots, \ldots, \geq b_{n}$ of nonnegative real numbers. The sequence $\{a\}$ is said to majorize the sequence $\{b\}$ if the following conditions (6) are fulfilled:

$$
\begin{aligned}
a_{1} \geq b_{1} ; a_{1}+a_{2} \geq b_{1}+b_{2} ; \ldots ; a_{1}+a_{2}+\ldots+a_{n-1} \geq b_{1}+b_{2}+\ldots+b_{n-1} ; \\
a_{1}+a_{2}+\ldots+a_{n-1}+a_{n}=b_{1}+b_{2}+\ldots+b_{n-1}+b_{n}
\end{aligned}
$$

For any set of non-negative numbers $x_{1}, x_{2}, \ldots, x_{n}$, a symmetric sum is defined as $\sum_{\text {sym }} x_{1}^{a_{1}} x_{2}^{a_{2}} \ldots x_{n}^{a_{n}}$ which, when expanded, includes $n$ ! terms. Each term is formed by a permutation of the elements of the sequence $a_{1}, a_{2}, \ldots, a_{n}$. Thus, if $\{a\}=[2,1,0]$ then

$\sum_{\text {sym }} x_{1}^{2} x_{2}^{1} x_{3}^{0}=x_{1}^{2} x_{2}+x_{1}^{2} x_{3}+x_{2}^{2} x_{1}+x_{2}^{2} x_{3}+x_{3}^{2} x_{1}+x_{3}^{2} x_{2}$

If $\{a\}=[2,0,0]$, then

$\sum_{\text {sym }} x_{1}^{2} x_{2}^{0} x_{3}^{0}=2 x_{1}^{2}+2 x_{2}^{2}+2 x_{3}^{2}$

Consider now the set of non-negative numbers $x_{1}, x_{2}, x_{3}$ and the sequences $\{a\}=[2,0,0]$ and $\{b\}=[1,1,0]$. Clearly, the sequence $\{a\}=[2,0,0]$ majorizes the sequence $\{b\}=[1,1,0]$ because the conditions (6) are fulfilled:

$2 \geq 1 ; 2+0 \geq 1+1$ and $2+0+0=1+1+0$.

According to the Muirhead's inequality (5):

$$
2 ! \times\left(x_{1}^{2}+x_{2}^{2}+x_{3}^{2}\right) \geq 2\left(x_{1} x_{2}+x_{1} x_{3}+x_{2} x_{3}\right)
$$

Dividing both sides of the last inequality by the positive constant 3 ! gives inequality (1).

Because the sequence $\{a\}=[3,0,0]$ majorizes the sequence $\{b\}=[1,1,1]$, the next inequality follows directly from the Muirhead's inequality (5):

$$
(n-1) ! \times\left(x_{1}^{3}+x_{2}^{3}+x_{3}^{3}\right) \geq n ! \times x_{1} x_{2} x_{3}
$$

is obtained.

By dividing both sides of (8) to $n !(n=3)$, inequality (8) transforms into inequality (3).

Since the sequence $\{a\}=[3,0,0]$ also majorizes the sequence $\{c\}=[2,1,0]$, the following inequality follows immediately from the Muirhead's inequality (5):

$$
(n-1) ! \times\left(x_{1}^{3}+x_{2}^{3}+x_{3}^{3}\right) \geq x_{1}^{2} x_{2}+x_{1}^{2} x_{3}+x_{2}^{2} x_{1}+x_{2}^{2} x_{3}+x_{3}^{2} x_{1}+x_{3}^{2} x_{2}
$$

Dividing both sides of (9) by 3 ! ( $n=3)$, gives inequality (4).

Note that the Muirhead inequality does not hold for the sequence $\{b\}=[0,3,0]$ because the requirement is non-increasing sequences $a_{1} \geq a_{2}, \ldots, \geq a_{n}$ and $b_{1} \geq b_{2}, \ldots, \ldots, \geq b_{n}$ of nonnegative real numbers and the sequence $\{b\}=[0,3,0]$ is not a non-increasing sequence.

All theoretical results from the application of the inequalities have been confirmed by Monte-Carlos simulations, each of which involved 10 million trials. For fractions of highreliability components $0.9,0.55$ and 0.35 charactering the separate suppliers, the Monte-Carlo simulation yielded probabilities $p_{1}=0.41$ and $p_{2}=0.33$ of purchasing two high-reliability components from a randomly selected single supplier and from two randomly selected suppliers, correspondingly. The left and right part of the inequality (1) give $p_{1}=0.41$ and $p_{2}=0.33$ for the same probabilities, which confirms the validity of the inequality (1).

The basic idea behind the simulation of purchasing two components from a randomly selected supplier and testing the components for high-reliability is done within a loop of ten 
million trials. The pseudo-code fragments related to evaluating the empirical probability of purchasing two high-reliability components from a single supplier and from two randomly selected suppliers are shown in the Appendix (Algorithm 1 and Algorithm 2).

For the probabilities of purchasing three high-reliability components from a randomly selected single supplier and from the three available suppliers the Monte-Carlo simulation resulted in probabilities $p_{1}=0.313$ and $p_{2}=0.17$, correspondingly. The left and right part of inequality (3) give $p_{1}=0.313$ and $p_{2}=0.17$ for the same probabilities, which confirms the validity of the derived inequality.

For the probabilities of purchasing three high-reliability components from a randomly selected single supplier and from two randomly selected suppliers, the Monte-Carlo simulation yielded $p_{1}=0.313$ and $p_{2}=0.2142$, correspondingly. The left and right side of inequality (4) give $p_{1}=0.313$ and $p_{2}=0.2141$ for the same probabilities, which confirms the validity of the derived inequality.

The Monte-Carlo simulation algorithms relevant to purchasing three components are very similar to the simulation algorithm described earlier and to conserve space, details have been omitted.

In summary, if no information is available about the components reliability characterising the separate suppliers, the best strategy is to purchase the components from a single supplier or from the smallest possible number of suppliers.

\section{USING INEQUALITIES FOR RANKING SYSTEMS WITH UNKNOWN RELIABILITIES OF THE COMPONENTS}

The first approach of using inequalities for reliability improvement and risk reduction can also be demonstrated with comparing the reliabilities of competing systems. It starts with building the functional diagram of the system, creating its reliability network, deriving expressions for the system reliability of the competing alternatives, conjecturing inequalities about the competing alternatives, testing the inequalities and if the inequalities are confirmed, proving the inequalities rigorously by using some combination of the listed analytical techniques for proving inequalities.

In the case where the reliabilities of the components are known, the performance of the competing systems can be compared through Monte Carlo simulations ${ }^{32}$. Often, this approach is not viable because the reliabilities of the components building the system are unknown. For two competing systems (a) and (b), the method based on inequalities for determining which system is superior in terms of reliability can be summarized by the following steps.

- Build the reliability networks of the competing systems from their functional diagrams.

- Determine the system reliabilities of the competing systems $R_{a}$ and $R_{b}$ or the probabilities of system failure $F_{a}$ and $F_{b}$ by constructing corresponding analytical expressions.

- Subtract the system reliabilities or the probabilities of failure and prove any of the inequalities: $R_{a}-R_{b}>0, R_{a}-R_{b}<0 \quad F_{a}-F_{b}>0, F_{a}-F_{b}<0$ regarding the system reliabilities or the probabilities of system failure.

- Select the system with the superior reliability.

\subsection{Ranking systems with the same topology and different components arrangements}

Consider the functional diagrams of three systems built with pipes and four valves $(A, B, C$ and $D$ ) with different ages (Fig.2). Valve $A$ is a new valve, followed by valves $B$ and $C$ with 
intermediate age of which valve $C$ is older than valve $B$. Valve $D$ is an old valve whose age is greater than the age of any other valve. The reliabilities of the valves are denoted by $a, b, c$ and $d$. With respect to the function 'valve closure on demand', depending on their age, the reliabilities of the valves can be ranked as follows: $a>b>c>d$. The valves are working independently from one another and initially, all of them are open. The question of interest is which system is more reliable with respect to the function 'stopping the fluid flow in the pipeline on command'. The signal for closing is issued to all valves simultaneously.

It has been conjectured that the arrangement in Figure $2 \mathrm{c}$ is superior to the arrangements in Figure $2 \mathrm{a}$ and $2 \mathrm{~b}$.

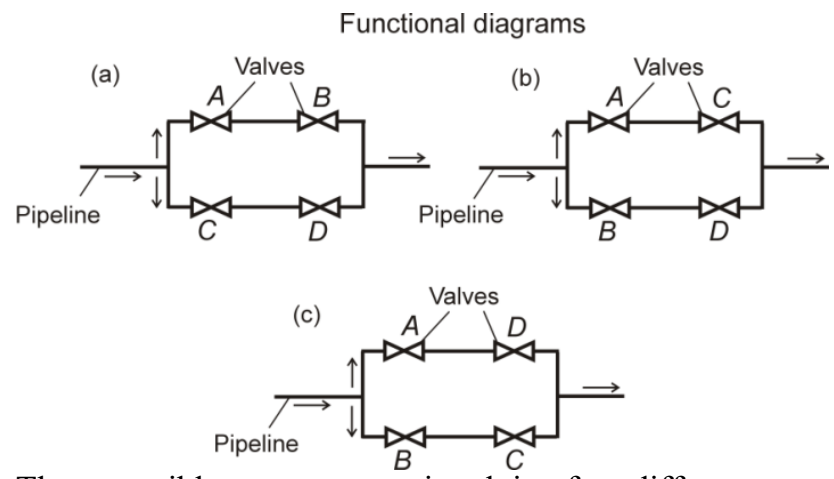

Figure 2. Three possible arrangements involving four different types of valves

The reliability networks (reliability block diagrams) of the systems with respect to the function 'stopping the fluid in the pipeline on command', are given in Fig.3.

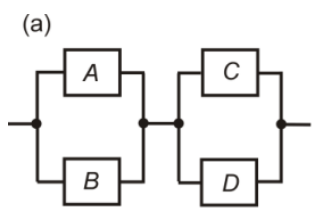

(b)

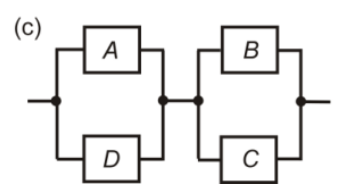

Figure 3. Reliability networks of the systems in Fig. 2 with respect to the function 'stopping the fluid in the pipeline on command.

The reliabilities of systems (a), (b) and (c) are given by

$$
\begin{aligned}
& R_{a}(a, b, c, d)=[1-(1-a)(1-b)] \times[1-(1-c)(1-d)]=(a+b-a b)(c+d-c d) \\
& R_{b}(a, b, c, d)=[1-(1-a)(1-c)] \times[1-(1-b)(1-d)]=(a+c-a c)(b+d-b d) \\
& R_{c}(a, b, c, d)=[1-(1-a)(1-d)] \times[1-(1-b)(1-c)]=(a+d-a d)(b+c-b c)
\end{aligned}
$$

Proving that the configuration in Figure $2 \mathrm{c}$ is superior reduces to proving the inequalities

$$
\begin{aligned}
& R_{c}(a, b, c, d)>R_{a}(a, b, c, d) \\
& R_{c}(a, b, c, d)>R_{b}(a, b, c, d)
\end{aligned}
$$

which are equivalent to the inequalities

$$
\begin{aligned}
& (a+d-a d)(b+c-b c)-(a+b-a b)(c+d-c d)>0 \\
& (a+d-a d)(b+c-b c)-(a+c-a c)(b+d-b d)>0
\end{aligned}
$$

Manipulating the left side of inequality (13) results in

$(a+d-a d)(b+c-b c)-(a+b-a b)(c+d-c d)=$ $a b+d c-a d-b c=a(b-d)-c(b-d)=(a-c)(b-d)$ 
Considering that $a>b>c>d$, it follows that $(a-c)(b-d)>0$. Therefore, the configuration in Figure 2c is more reliable than the configuration in Figure 2a.

Manipulating the left hand side of inequality (14) results in

$(a+d-a d)(b+c-b c)-(a+c-a c)(b+d-b d)=$

$a c+d b-a d-c b=a(c-d)-b(c-d)=(a-b)(c-d)$

Considering that $a>b>c>d$, it follows that $(a-b)(c-d)>0$. Therefore, the configuration in Figure $2 \mathrm{c}$ is more reliable than the configuration in Figure 2b. Consequently, the configuration in Figure 2c is the most reliable configuration.

\subsection{Ranking systems with different topologies built with the same type of components}

Often, the reliabilities of the components building the system or the ages of the components are unknown and the epistemic uncertainty associated with the reliability ranking of the components building the system translates into epistemic uncertainty related to which system is superior. To demonstrate that this case too can be handled by using algebraic inequalities, two systems with different topologies including the same type of valves are shown in Figure 4. The reliabilities or the age of the valves are unknown. The valves are working independently from one another and all of them are initially open. The question of interest is which system is more reliable with respect to the function 'stopping the fluid flow in the pipeline'. The signal for closing is issued to all valves simultaneously.

\section{Functional diagrams}

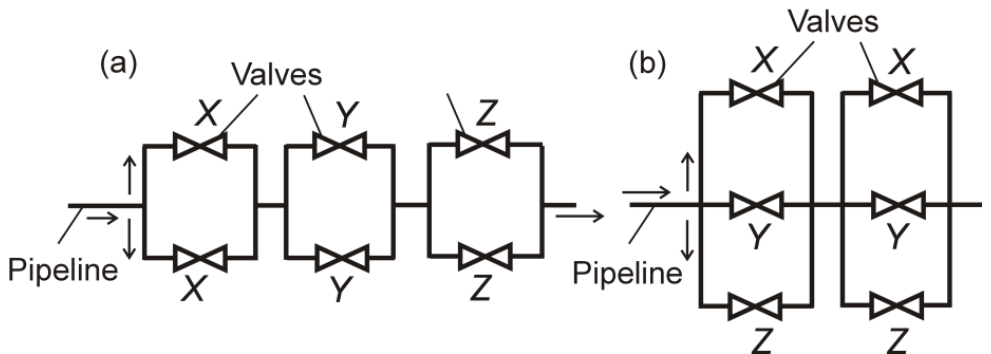

Figure 4. Two competing systems with different topology, built with the same type and number of components.

a)

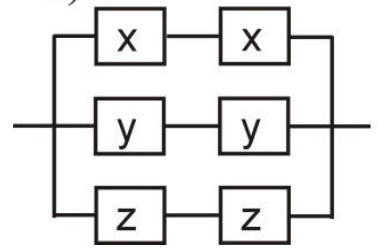

b)

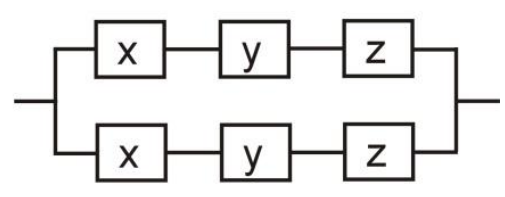

Figure 5. The reliability networks of the systems from Figure 4.

The reliability networks of the systems from Fig.4 are shown in Fig.5. There are three types of independently working valves whose reliabilities are denoted by $x, y$ and $z$. The reliability values $x, y$ and $z$ characterising the separate valves are unknown. The only available information about the reliabilities of the valves are the obvious constraints: $0<x<1 ; 0<y<1 ; 0<z<1$. Expressing the probabilities of failure characterising the competing systems as a function of the unknown reliabilities of the valves yields:

$F_{a}(x, y, z)=\left(1-x^{2}\right)\left(1-y^{2}\right)\left(1-z^{2}\right)$ and $F_{b}(x, y, z)=(1-x y z)^{2}$. 
Ranking the system reliabilities consists of proving $F_{a}(x, y, z)-F_{b}(x, y, z)<0$ or $F_{a}(x, y, z)-F_{b}(x, y, z)>0$. Proving $F_{a}(x, y, z)-F_{b}(x, y, z)<0$ for example, is equivalent to proving the inequality

$$
\left(1-x^{2}\right)\left(1-y^{2}\right)\left(1-z^{2}\right)<(1-x y z)^{2}
$$

To prove inequality (15) it suffices to prove the inequality $\sqrt{\left(1-x^{2}\right)\left(1-y^{2}\right)\left(1-z^{2}\right)}<(1-x y z)$ or the equivalent inequality

$$
\sqrt{\left(1-x^{2}\right)\left(1-y^{2}\right)\left(1-z^{2}\right)}+x y z<1
$$

Indeed, if inequality (16) is true, inequality (15) follows from it by squaring both sides of the inequality $\sqrt{\left(1-x^{2}\right)\left(1-y^{2}\right)\left(1-z^{2}\right)}<1-x y z$. The squaring operation will not change the direction of the inequality because $0<x<1 ; 0<y<1 ; 0<z<1$, and the quantities:

$$
(1-x y z)>0,\left(1-x^{2}\right)>0 ;\left(1-y^{2}\right)>0 ;\left(1-z^{2}\right)>0 \text { are all positive. }
$$

To prove inequality (16), a combination of the 'substitution' technique and 'proving a simpler, intermediate inequality' technique will be used.

Because the reliability of a component is a number smaller than unity, trigonometric substitutions $r_{i}=\sin \alpha_{i}$ where $\alpha_{i} \in(0, \pi / 2)$ are appropriate.

Making the substitutions: $x=\sin \alpha ; y=\sin \beta$ and $z=\sin \gamma$ transforms the left side of inequality (16) into

$$
\sqrt{\left(1-x^{2}\right)\left(1-y^{2}\right)\left(1-z^{2}\right)}+x y z=\cos \alpha \times \cos \beta \times \cos \gamma+\sin \alpha \times \sin \beta \times \sin \gamma
$$

Next, the positive quantity $\cos \alpha \times \cos \beta \times \cos \gamma+\sin \alpha \times \sin \beta \times \sin \gamma$ is replaced by the larger quantity $\cos \alpha \times \cos \beta+\sin \alpha \times \sin \beta$. Indeed, because $0<\cos \gamma<1$ and $0<\sin \gamma<1$, the inequality

$$
\cos \alpha \times \cos \beta \times \cos \gamma+\sin \alpha \times \sin \beta \times \sin \gamma<\cos \alpha \times \cos \beta+\sin \alpha \times \sin \beta
$$

holds.

If the intermediate inequality $\cos \alpha \times \cos \beta+\sin \alpha \times \sin \beta \leq 1$ can be proved, the inequality (16) will follow immediately from inequality (17).

Since $\cos \alpha \times \cos \beta+\sin \alpha \times \sin \beta=\cos (\alpha-\beta)$, and $\cos (\alpha-\beta) \leq 1$, we finally get

$\cos \alpha \times \cos \beta \times \cos \gamma+\sin \alpha \times \sin \beta \times \sin \gamma<\cos \alpha \times \cos \beta+\sin \alpha \times \sin \beta=\cos (\alpha-\beta) \leq 1$

from which inequalities (16) and (15) follow. The system in Figure 4a is characterised by a smaller probability of failure compared to the system in Figure $4 b$, therefore, the system in Figure $4 \mathrm{a}$ is intrinsically more reliable than the system in Figure $4 \mathrm{~b}$.

\subsection{Confirming or disproving an inequality by a Monte-Carlo simulation}

Before attempting to prove a derived or conjectured inequality rigorously, it is important to confirm it first by testing it by simulation. An attempt is made to prove the inequality rigorously only if the simulation provides support for the conjectured inequality. No attempt is made to prove the inequality rigorously if, during the simulation, the inequality has been falsified by a counterexample.

Suppose that $n$ distinct components with unknown reliabilities $a_{1}, a_{2}, \ldots, a_{n}$ are used for building two systems with reliabilities $f\left(a_{1}, \ldots, a_{n}\right)$ and $g\left(a_{1}, \ldots, a_{n}\right)$. To test the conjecture that the system topology with reliability $f\left(a_{1}, \ldots, a_{n}\right)$ is intrinsically superior to the system topology with reliability $g\left(a_{1}, \ldots, a_{n}\right)$, irrespective of the specific reliabilities $a_{1}, a_{2}, \ldots, a_{n}$ of the components building both systems, it suffices to test that the inequality 
$f\left(a_{1}, \ldots, a_{n}\right)>g\left(a_{1}, \ldots, a_{n}\right)$ is not contradicted during the simulation trials. This can be done by running Algorithm 3 in the Appendix, given in pseudo-code.

In general, the variables $a_{i}$ entering in the inequality $f\left(a_{1}, \ldots, a_{n}\right)>g\left(a_{1}, \ldots, a_{n}\right)$ to be tested vary within specified intervals $L_{i} \leq a_{i} \leq U_{i}, i=1, \ldots, n$. If $a_{i}$ are reliabilities of the components then $L_{i}=0, U_{i}=1$.

The essence of Algorithm 3 in the Appendix for testing a conjectured inequality is repeated sampling from the intervals of variation of each variable $a_{i}$ entering the inequality, substituting the sampled values in the inequality and checking whether the inequality $f\left(a_{1}, \ldots, a_{n}\right)>g\left(a_{1}, \ldots, a_{n}\right)$ holds. Even a single combination of values for the variables $a_{i}$, for which the inequality does not hold, disproves the inequality and shows that there is no point in attempting a rigorous proof because a counterexample has been found which falsified the conjectured inequality.

If the inequality holds for millions of generated random values for the sampled variables, a strong support is obtained for the conjecture that the tested inequality is true. However, such support cannot replace a rigorous proof. The tested inequality must be proved rigorously by using some combination of techniques for proving inequalities.

\section{MEANINGFUL INTERPRETATION OF ABSTRACT INEQUALITIES TO IMPROVE RELIABILITY AND REDUCE UNCERTAINTY AND RISK}

There is an alternative approach of using algebraic inequalities for modelling real systems and processes. This method consists of moving in the opposite direction: starting with an existing abstract inequality and moving towards the real system or process. A key step of this very powerful approach is creating relevant meaning for the variables entering the algebraic inequality, followed by a meaningful interpretation of the left-and right-side of the inequality (Figure 6)

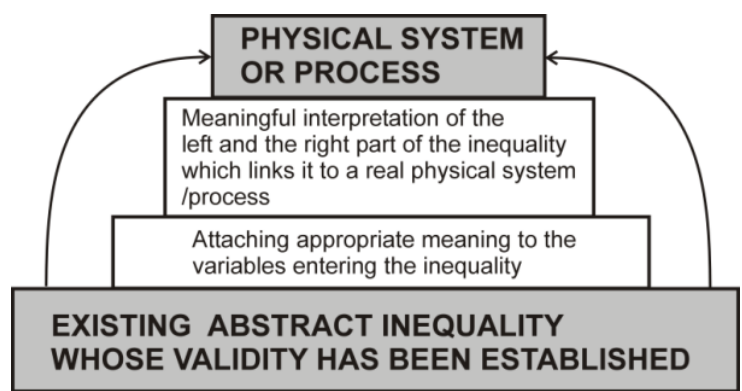

Figure 6. Reducing risk and uncertainty and optimising systems performance by creating relevant meaning and interpretation for existing abstract inequalities.

This process effectively links the abstract algebraic inequality with a real physical system or process and not only opens opportunities for enhancing the performance of systems and processes but also leads to the discovery of new fundamental properties of systems and processes. The key parts of the process of meaningful interpretation of an algebraic inequality are: (i) in various specific domains identifying classes of abstract inequalities which permit a meaningful interpretation that can be linked with a real system or process; (ii) identifying various transformations of known inequalities which make their meaningful interpretation possible in the listed domains; (iii) creating relevant meaning for the variables entering the algebraic inequality and (iv) meaningful interpretation of the different parts of the inequality and linking it into a real physical system or process. 


\subsection{Meaningful interpretation of a special case of the Cauchy-Schwarz inequality related to equivalent properties of elements connected in series and parallel}

Consider an example of this approach related to obtaining tight bounds for properties. The abstract algebraic inequality (18)

$$
\left(x_{1}+x_{2}+\ldots+x_{n}\right) \geq n^{2}\left(\frac{1}{1 / x_{1}+1 / x_{2}+\ldots+1 / x_{n}}\right)
$$

is valid for any set of $n$ non-negative quantities $x_{i}$. This inequality can be proved rigorously by using the technique 'reducing to a standard inequality' listed in the introduction. The inequality can be proved rigorously by reducing it to the standard Cauchy-Schwarz inequality $^{12}$, which states that for any two sequences of real numbers $\left\{a_{1}, a_{2}, \ldots, a_{n}\right\}$ and $\left\{b_{1}, b_{2}, \ldots, b_{n}\right\}$, the following sharp inequality holds:

$$
\left(a_{1} b_{1}+a_{2} b_{2}+\ldots+a_{n} b_{n}\right)^{2} \leq\left(a_{1}^{2}+a_{2}^{2}+\ldots+a_{n}^{2}\right)\left(b_{1}^{2}+b_{2}^{2}+\ldots+b_{n}^{2}\right)
$$

Equality holds if and only if for any $i \neq j, a_{i} b_{j}=a_{j} b_{i}$ are fulfilled.

Because the quantities $x_{i}$ are non-negative, the setting $a_{i}=\sqrt{x_{i}} ; i=1, n$ and $b_{i}=1 / \sqrt{x_{i}} ; i=1, n$ can be made. Next, substituting $a_{i}$ and $b_{i}$ in the Cauchy-Schwarz inequality (19) gives the inequality (18).

In order to make an existing abstract inequality relevant to reliability and risk, appropriate meaning must be attached to the variables entering the inequality.

Suppose that the variables $x_{i}$ in inequality (18) stand for stiffness of an elastic element $i$. Now, the two sides of the inequality can be meaningfully interpreted in the following way. The expression $x_{1}+x_{2}+\ldots+x_{n}$ on the left hand side of the inequality (18) can be interpreted as the equivalent elastic constant for $n$ elastic elements arranged in series. The right side $\frac{1}{1 / x_{1}+1 / x_{2}+\ldots+1 / x_{n}}$ of the inequality can be interpreted as the equivalent elastic constant for $n$ elastic elements arranged in parallel. The inequality can be interpreted as a comparison of the equivalent stiffness of $n$ elastic elements arranged in series (the left side) with the equivalent stiffness of the same $n$ elastic elements arranged in parallel (the right side). The inequality expresses a physical law: the equivalent stiffness of $n$ elements arranged in series is at least $n^{2}$ times larger than the equivalent stiffness of the same elements arranged in parallel, irrespective of the individual stiffness values of the elements.

The meaning created for the variables in the inequality is not unique and can be altered. Thus, a new relevant meaning for the variables can be created, for example each $x_{i}$ now stands for "electrical resistance of element $i$ ".

The expression $x_{1}+x_{2}+\ldots+x_{n}$ on the left side of the inequality can now be meaningfully interpreted as the equivalent resistance of $n$ resistors arranged in series. On the right side of the inequality, the expression $\frac{1}{1 / x_{1}+1 / x_{2}+\ldots+1 / x_{n}}$ can be interpreted as the equivalent resistance of $n$ resistors arranged in parallel. Inequality (18) now expresses another physical law: the equivalent resistance of $n$ resistors arranged in series is at least $n^{2}$ times larger than the equivalent resistance of the same resistors arranged in parallel, irrespective of the individual values of the resistors. 
Suppose that to provide a reliable insulation from a high-voltage source and safe operation, the equivalent resistance $R_{s}=x_{1}+x_{2}+\ldots+x_{n}$ of $n$ elements connected in series must be larger than a minimum threshold safe value $T_{R}\left(R_{s} \geq T_{R}\right)$. The knowledge created from the meaningful interpretation of inequality (18) can be used to test whether the equivalent resistance $R_{s}$ of $n$ elements with very large resistances, connected in series, is greater than the safe threshold value $T_{R}$. Measurement of very large resistances is often very difficult due to the unavailability of equipment capable of measuring such resistances. If the significantly smaller equivalent resistance $R_{p}$ of all of the elements connected in parallel is measured instead, a conclusion about the equivalent resistance of the same elements arranged in series can be made from the inequality $R_{s} \geq n^{2} R_{p}$ proved earlier. If the product of the measured resistance $R_{p}$ and $n^{2}$ is greater than the specified threshold: $n^{2} R_{p} \geq T_{R}$, then the safety requirement has been met and the risk of failure has been reduced.

It needs to be pointed out that for resistors of equal values, the fact that the equivalent resistance in parallel is exactly $n^{2}$ times smaller than the equivalent resistance of the resistors in series is a trivial result, easily derived and known for a long period of time.

Indeed, for the resistance of $n$ resistors arranged in series $x_{1}=x_{2}=\ldots=x_{n}=r$, the value $n r$ is obtained while for the same $n$ resistors arranged in parallel, the value $r / n$ is obtained. As can be seen, the value $r / n$ is exactly $n^{2}$ times smaller than the value $n r$. However, the bound provided by inequality (18) is a much deeper result. It is valid for any possible values of the resistances. The bound given by inequality (18) does not require equal resistances.

Now suppose that $x_{i}$ means capacitance. The expression $x_{1}+x_{2}+\ldots+x_{n}$ on the left side of inequality (18) can be meaningfully interpreted as the equivalent capacitance of $n$ capacitors arranged in parallel. On the right side of the inequality, the expression $\frac{1}{1 / x_{1}+1 / x_{2}+\ldots+1 / x_{n}}$ can be meaningfully interpreted as the equivalent capacitance of $n$ capacitors arranged in series. The inequality now expresses another physical law: the equivalent capacitance of $n$ capacitors arranged in parallel is at least $n^{2}$ times larger than the equivalent capacitance of the same capacitors arranged in series, irrespective of the values of the individual capacitors.

The proposed technique for reducing uncertainty based on meaningful interpretation of the Cauchy-Schwarz inequality is domain-independent. It transcends mechanical engineering and can be applied in many unrelated domains.

If multiple real experiments are performed in arranging elastic elements, resistors and capacitors in series and parallel and measuring the equivalent elastic constants resistance and capacity, the observations will not contradict inequality (18). Clearly, the statement expressed by inequality (18) and the behavior of the systems of springs, resistors, capacitors and thermal elements arranged in series and parallel are consistent and no contradiction will be present between the interpretation of the inequality and the behavior of the physical systems.

The considered examples illustrate physical properties predicted from interpreting a correct algebraic inequality and the principle of non-contradiction: if the variables and the different parts of an abstract inequality can be interpreted as parts of a physical process, in the real world, the physical process must take course such that it stays consistent with the predictions from the abstract inequality.

In other words, the realization of the process yields results that do not contradict the algebraic inequality. More details about the principle of non-contradiction will be presented elsewhere. 


\subsection{Meaningful interpretation of an algebraic inequality related to ranking the magnitudes of sequential random events}

This example of meaningful interpretation of an algebraic inequality has been prompted by a short note published in [34]. Consider the algebraic inequality

$$
p^{2}+(1-p)^{2}+4 p(1-p) \geq 1
$$

where $0 \leq p \leq 1$. This inequality can be proved easily by simplifying the left side to $1+2 p(1-p)$ which is obviously not smaller than one because $p(1-p) \geq 0$. If both sides of Inequality (20) are divided by 2 , the next equivalent inequality is obtained:

$$
\frac{1}{2} p^{2}+\frac{1}{2}(1-p)(1-p)+(1-p) p+p(1-p) \geq 1 / 2
$$

Inequality (21) can be meaningfully interpreted by creating meaning for the variable $p$ and for the left and right side of the inequality. Because $0 \leq p \leq 1$, it is natural to interpret the variable $p$ as 'probability'. Suppose that the variable $p$ stands for the probability that the magnitude of a random event exceeds a selected threshold $C$ (Figure 7a). The first term $\frac{1}{2} p^{2}$ in The first term $(1 / 2) p^{2}$ in inequality (21) can then be interpreted as the probability that the magnitudes of two sequential random events will both exceed the selected threshold $C$ and the second random event will have a smaller magnitude than the first random event (Figure $7 \mathrm{~b}$ ). The second term $\frac{1}{2}(1-p)(1-p)$ can be interpreted as the probability that the magnitudes of two future sequential random events will both be below the selected threshold $C$ and the second random event will have a larger magnitude than the first random event (Figure 7c). The third term $1 \times(1-p) p$ can be interpreted as the probability that the first random will be below the threshold $C$, the second random event will be above the threshold $C$ and will have a larger magnitude than the second event (Figure 7d). Finally, the term $1 \times p(1-p)$ can be interpreted as the probability that the first random event will be above the threshold $C$, the second random event will be below the threshold $\mathrm{C}$ and will have a smaller magnitude than the first event (Figure 7e).

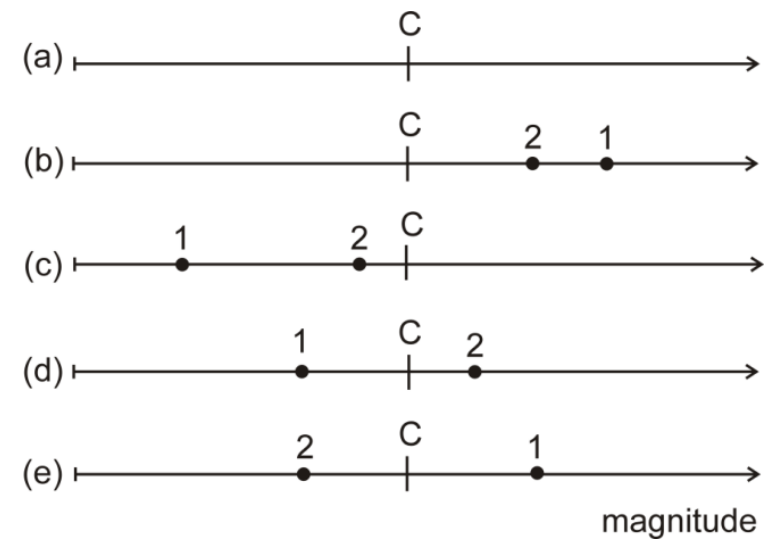

Figure 7. The random threshold $C$ increases the likelihood of a correct prediction of the magnitude ranking of future random events, to more than $50 \%$

Consider now a real-world problem related to estimating the probability that the magnitude of a future random event will be exceeded by the magnitude of the next random event. This is a problem related to predicting magnitude ranking of safety-critical random events, important in cases where it is necessary to decide on whether there is a need for extra resources to 
mitigate the consequences associated with future random events. Such random events could be, for example, the leaks of crude oil into marine water from a pipeline during subsea oil and gas production, the magnitudes of floods in consecutive years, the magnitudes of fires, the direction of movement of the price of a commodity on the stock market, etc. If no information is available about the distribution of the magnitude of the random event, it seems that the chance of guessing correctly whether the magnitude of the second random event will be larger than the magnitude of the first random event is only $50 \%$ and cannot be improved. Surprisingly, by introducing a random comparison threshold $C$ (before the occurrence of any random event, see Figure 7a), the uncertainty can be reduced and the chance of predicting correctly whether the magnitude of the second random event will exceed the magnitude of the first random event can be increased beyond $50 \%$.

The prediction strategy is simple [34]. If the magnitude of the first random event is larger than the randomly pre-selected comparison threshold $C$ (Figure $7 \mathrm{a}$ ) a prediction is made that the second random event will have a smaller magnitude than the magnitude of the first random event. If the magnitude of the first random event is smaller than the comparison threshold $C$, a prediction is made that the second random event will have a larger magnitude than the magnitude of the first event (Figure 7c).

In the cases where both random events have larger magnitudes than the pre-selected threshold $C$, or both random events have smaller magnitudes than the pre-selected threshold $C$, the likelihood of a correct prediction related to the magnitude of the second random event is clearly 0.5 . In the case where the first random event has a magnitude smaller than the threshold $C$ and the second event has a larger magnitude, the likelihood of a correct prediction related to the magnitude of the second random event is 1.0. Similarly, in the case where the first random event has a larger magnitude than the pre-selected threshold and the second event has a smaller magnitude, the likelihood of a correct prediction related to the magnitude of the second random event is also 1.0 .

Contrary to the statement made in [34], from inequality (21) it follows that the prediction strategy described earlier does not ensure correct prediction with probability strictly greater than 0.5 .

Indeed, if the threshold $C$ has been selected to be too large or too small within the physically possible range of values for the magnitude of the random event then the selected threshold grossly underestimates or overestimates the expected magnitude and $1-p \approx 0$ or $p \approx 0$. In this case, the quantity $p(1-p) \approx 0$ on the left side of $(21)$ is very small and practically, a probability of correct prediction beyond 0.5 cannot be attained. The probability of correct prediction cannot be increased also if, for example, the distribution of the magnitudes of the random event is characterised by a very small variance. In this case, the likelihood that the magnitudes of the two sequential random events will fall on both sides of the selected threshold $C$ is very small and again, $p(1-p) \approx 0$.

The product of two positive values with a given sum has a maximum when the values are equal. Consequently, the product $p q$ where $p+q=1$ attains maximum when $p=q=1 / 2$. As a result, the maximum possible value of the quantity $p(1-p)$ is 0.25 and this value cannot be exceeded. Consequently, the most appropriate position of the threshold $C$ is at a value for which the probability that the magnitude of the event will be smaller than the threshold is equal to the probability that the magnitude of the event will be larger than the threshold. In this case, a correct prediction will be made in $75 \%$ of the time.

In reality, the threshold position of equal probability is not known and the threshold is selected randomly within the physically possible range of values for the magnitude $M$.

To test the described decision strategy, a Monte-Carlo simulation experiment has been made. Suppose that the leak of crude oil is from a pipeline with a maximum debit of $300 \mathrm{l} / \mathrm{s}$. 
Because no leak can be negative and no oil leak can exceed the maximum debit of the pipeline, the lower and upper limit of the range of all possible leaks are abs_lower_limit=0; and abs_upper_limit $=300$, correspondingly. The possible leak magnitudes within the physically possible range are uncertain, this is why the distribution of the leak magnitude is assumed to be uniform, with randomly selected lower and upper limit $a$ and $b$ over the possible physical range $[0,300]$.

The algorithm testing the described decision strategy in pseudo-code is given in the Appendix (Algorithm 4).

Series of Monte-Carlo simulations each consisting of 10 million random trials resulted in probabilities of a correct prediction within the range [0.50 - 0.64], which confirmed that the proposed strategy indeed reduces uncertainty and yields a prediction result related to the magnitude ranking of the second leak which is better than a random guess. If the prediction is made randomly, without a comparison with a preliminarily selected threshold, the probability of a correct prediction is exactly $50 \%$.

If a new meaning is attached to the event, the abstract inequality (21) underlying the decision process for leaks can be applied to other real processes: movement of a stock price, magnitudes of random shocks on a device or structure, etc. Thus, the described technique for reducing uncertainty can also be used to transform a neutral bet with expected profit zero into a good bet with positive expected profit. A large number of good bets materialises into gain ${ }^{33}$.

\subsection{Meaningful interpretation of sub-additive and super-additive inequalities related to properties expressed as power laws}

Apart from the considered inequalities, there exist wider classes of algebraic inequalities which can be used to attain superior performance through meaningful interpretation. Inequalities based on sub-additive and super-additive functions are such examples.

Consider a factor $x$ that has been segmented into a number of non-negative parts $x_{1}, x_{2}, \ldots, x_{n}\left(x=x_{1}+x_{2}+\ldots+x_{n}\right)$. The output associated with the factor $x$ and each of the segments $x_{i}$ is given by the function $f()$. The output function $f()$ is said to be sub-additive if, for any sequence of real numbers $x_{1}, x_{2}, \ldots, x_{n}$, the next inequality holds:

$$
f\left(x_{1}+x_{2}+\ldots+x_{n}\right) \leq f\left(x_{1}\right)+f\left(x_{2}\right)+\ldots+f\left(x_{n}\right)
$$

The output function $f\left(0\right.$ is super-additive, if for any sequence $x_{1}, x_{2}, \ldots, x_{n}$, the next inequality holds:

$$
f\left(x_{1}+x_{2}+\ldots+x_{n}\right) \geq f\left(x_{1}\right)+f\left(x_{2}\right)+\ldots+f\left(x_{n}\right)
$$

Note that if the output function $f()$ is linear, equality is attained in inequalities (22) and (23). Multiplying the definition inequalities (22) and (23) by '-1', reverses the direction of the inequalities. Consequently, $f()$ is sub-additive if and only if $-f()$ is super-additive and $f()$ is super-additive if and only if $-f()$ is sub-additive. An example of a super-additive output function defined for $0 \leq x<\infty$ is the function $e^{x}$.

Key results related to sub-additive and super-additive functions have been stated in [35]. Thus, if a function $f()$, with a domain $[0, \infty)$ and range $[0, \infty)$ is concave, and $f(0) \geq 0$ then the function is sub-additive: $f\left(x_{1}+x_{2}+\ldots+x_{n}\right) \leq f\left(x_{1}\right)+f\left(x_{2}\right)+\ldots+f\left(x_{n}\right)$. If the function $f()$ is convex and $f(0) \leq 0$, then it is super-additive: $f\left(x_{1}+x_{2}+\ldots+x_{n}\right) \geq f\left(x_{1}\right)+f\left(x_{2}\right)+\ldots+f\left(x_{n}\right)$. 
The meaningful interpretation of inequalities (22) and (23) can be applied for optimising processes. If the function $f(\bullet)$ reflects the effect/output of a particular factor and $x_{i}$ denote the different magnitudes of the factor, inequalities (22) and (23) provide the unique opportunity to increase the effect of the factor by segmenting or aggregating it, depending on whether the function $f()$ is concave or convex. If the function $f()$ is concave, with a domain $[0, \infty)$ and range $[0, \infty)$, segmenting the factor results in a larger output. Conversely, if the function $f()$ is convex in this domain, aggregating the factor results in a larger output. The knowledge about whether the function is sub-additive or super-additive is sufficient to reduce uncertainty.

An important condition for using sub-additive or super-additive inequalities is that the outputs $f\left(x_{1}\right), f\left(x_{2}\right), \ldots, f\left(x_{n}\right)$ after segmenting the control factor $x$ must be additive. This is fulfilled if the outputs $f\left(x_{1}\right), f\left(x_{2}\right), \ldots, f\left(x_{n}\right)$ are energy, power, force, damage, profit, pollution, mass, number, volume, numbers, etc.

The use of sub-additive and super-additive inequalities in process optimisation will be illustrated by power-type dependences involving a controlled factor. Power functions are widespread. Many real phenomena are approximated very well by a power-law. Power laws appear, for example, in cases where positive feedback loops are determining the output. Often, at the heart of the positive feedback loops is the preferential attachment, according to which a commodity is distributed according to how much commodity is already present.

Many real phenomena are approximated very well by power functions of the type

$$
y=a x^{p}
$$

where $a, p$ are constants $(a \neq 0 ; p>0), x$ is the controlling factor $\left(x \geq x_{0}\right)$ and $y$ is the output quantity.

The power functions expressed by equation (24) are encountered frequently in models of various phenomena, where $y$ stands for energy, power, force, damage, profit, pollution, etc.

Depending on whether the power $p$ is greater or smaller than unity, sub-additive or superadditive inequalities can be used to perform segmentation or aggregation of the controlling factor in order to attain enhanced performance.

The output quantity $y$ can be a convex function of the controlling factor $x(x \geq 0)$ depending on whether the second derivative $d^{2} y / d x^{2}=a p(p-1) x^{p-2}$ with respect to $x$ is positive or negative. This depends on whether the power $p$ is greater or smaller than 1 . If $p>1$, $d^{2} y / d x^{2} \geq 0$, and the power function $y$ is convex. If $0<p<1, d^{2} y / d x^{2} \leq 0$, and the power function $y$ is concave. If $p<0, d^{2} y / d x^{2}=a p(p-1) x^{p-2}>0$ and the power function $y$ is convex.

Suppose that the output function $y$ is of the type presented by equation (24). For a controlling factor $x$ varying in the interval $[0, \infty)$ and output quantity $y$ varying within the range $[0, \infty)$, the function (24) is convex if $p>1$ and since $y(0)=0$, the following super-additive inequality holds:

$$
a\left(x_{1}+x_{2}+\ldots+x_{n}\right)^{p} \geq a x_{1}^{p}+a x_{2}^{p}+\ldots+a x_{n}^{p}
$$

This inequality means that aggregating the controlling factor $x$ into $n$ parts yields a larger total effect.

If $0<p<1$ the power function (24) is concave and the following sub-additive inequality holds:

$$
a\left(x_{1}+x_{2}+\ldots+x_{n}\right)^{p} \leq a x_{1}^{p}+a x_{2}^{p}+\ldots+a x_{n}^{p}
$$

which means that segmenting the controlling factor $x$ yields a larger total effect. 
Consider an application example involving elastic strain energy accumulated by elastic elements. Suppose that the elastic strain energy accumulated due to a displacement $x$ is given by the power function:

$$
U=a x^{p}
$$

where $a$ and $p(p>1)$ are constants which depend on the material and its elastic properties. The controlling factor in the power-law dependence (27) is the displacement $x$ of the loaded elastic element.

For $p>1$, the quantity $U$ of accumulated elastic strain energy is a convex function of the displacement because the second derivative with respect to the displacement $x$ is positive: $d^{2} U / d x^{2}=a p(p-1) x^{p-2}>0$. Because for a displacement varying in the interval $[0, \infty)$, the quantity of elastic strain energy is within the range $[0, \infty)$ and $U(0)=0$, the function (27) giving the elastic strain energy is super-additive. Consequently, the inequality (25) holds.

The right side of inequality (25) can be interpreted as the sum of the elastic strain energy accumulated in $n$ elastic elements subjected to displacements $x_{1}, x_{2}, \ldots, x_{n}$ while the left side can be interpreted as quantity of potential energy accumulated in a single elastic element subjected to displacement equal to the sum of the displacements $x_{1}, x_{2}, \ldots, x_{n}$ of the separate elastic elements.

As a result, the interpretation of inequality (25) leads to an interesting conclusion: The elastic energy stored in $n$ elastic elements with displacements $x_{1}, x_{2}, \ldots, x_{n}$, respectively, is smaller than the elastic energy stored in a single elastic element with displacement of magnitude $x$, equal to the sum $x=x_{1}+x_{2}+\ldots+x_{n}$ of the displacements of multiple elastic elements.

Consider a non-linear, fully elastic element where the loading force $F$ varies with the displacement $\delta$, according to the dependence:

$$
F(\delta)=a \sqrt{\delta}
$$

where $a>0$ is a material constant. The accumulated elastic energy if the displacement varies within the limits $0 \leq \delta \leq x$ is given by

$$
U(x)=\int_{0}^{x} F(\delta) d \delta=\int_{0}^{x} a \sqrt{\delta} d \delta=(2 / 3) a x^{3 / 2}
$$

Because $p>1$ in (27), inequality (25) becomes:

$$
(2 / 3) a\left(x_{1}+x_{2}+\ldots+x_{n}\right)^{3 / 2} / 2 \geq(2 / 3) a x_{1}^{3 / 2}+(2 / 3) a x_{2}^{3 / 2}+\ldots+(2 / 3) a x_{n}^{3 / 2}
$$

The interpretation of inequality (29) leads to an interesting conclusion: the elastic strain energy accumulated into a single elastic element compressed at a displacement $x$ is greater than the elastic strain energy accumulated into $n$ elastic elements identical to the original elastic element, compressed to displacements $x_{1}, x_{2}, \ldots, x_{n} \quad\left(x=x_{1}+x_{2}+\ldots+x_{n}\right)$ whose sum is equal to the displacement of the original elastic element.

A similar type of inequality is, of course, valid for a linear elastic element with stiffness $k$. In this case, $a=k / 2 ; p=2$ and the expression for the elastic strain energy becomes $U=k x^{2} / 2$ . Inequality (25) becomes

$$
k\left(x_{1}+x_{2}+\ldots+x_{n}\right)^{2} / 2 \geq k x_{1}^{2} / 2+k x_{2}^{2} / 2+\ldots+k x_{n}^{2} / 2
$$

Consider now another application, in business. Suppose that the annual profit $z$ from an investment in a particular enterprise is given by the power function:

$$
z=c x^{q}
$$


where $x$ is the size of the investment, $c$ and $q(q<1)$ are constants which depend on the particular enterprise. The controlling factor in the power-law dependence (31) is the size of the investment $x$.

For $0<q<1$, the profit $z$ is a concave function of the size of investment because the second derivative with respect to the displacement $x$ is negative: $d^{2} z / d x^{2}=c q(q-1) x^{q-2}$. Because for investment varying in the interval $[0, \infty)$, the profit varies in the range $[0, \infty)$ and $z(0)=0$, the function (31) giving the profit is sub-additive. Consequently, inequality (26) becomes

$$
c\left(x_{1}+x_{2}+\ldots+x_{n}\right)^{q} \leq a x_{1}^{q}+a x_{2}^{q}+\ldots+a x_{n}^{q}
$$

The interpretation of inequality (32) leads to an important conclusion: splitting the initial investment $x$ and investing in $n$ parallel enterprises yields more total profit than investing the entire sum $x$ in a single enterprise. The difference in profit can be significant as can be verified from the next numerical example. For a profit dependence

$$
z=15.3 x^{0.42}
$$

the profit from investing $x=\$ 10000$ is $\$ 732.3$. Splitting the investment in two and investing $\$ 5000$ in two parallel enterprises gives $z=2 \times\left(15.3 \times 5000^{0.42}\right)=\$ 1094.7$ which is by $50 \%$ bigger than the profit from the initial investment.

The proposed technique for reducing uncertainty and risk based on meaningful interpretation of sub-additive and super-additive inequalities is domain-independent. It transcends mechanical engineering and can be applied for risk and uncertainty reduction in many unrelated domains.

\section{CONCLUSIONS}

Two fundamental approaches for reliability improvement and risk reduction by using nontrivial algebraic inequalities have been introduced: (i) by proving an algebraic inequality conjectured from a real system or process and (ii) by creating relevant meaning for an existing abstract inequality.

A formidable advantage of the algebraic inequalities can be found in their capacity to produce tight bounds related to reliability-critical design parameters, in the absence of any knowledge about the variation of the controlling variables. In this respect, the effectiveness of the first approach has been demonstrated by examples related to ranking of decision alternatives in the absence of sufficient detail and ranking of systems built on components whose reliabilities are unknown. By using inequalities, a highly counter-intuitive result has been obtained. If no information related the proportion of high-reliability components characterising the individual suppliers is available, purchasing components from a single supplier or from the smallest possible number of suppliers maximises the probability of a high-reliability assembly.

As part of the second approach, meaningful interpretation has been created for an inequality obtained as a special case of the Cauchy-Schwarz inequality. By varying the interpretation of the variables, the same inequality has been be applied for elastic elements, resistors and capacitors arranged in series and parallel.

Meaningful interpretation of super-additive and sub-additive inequalities have been used with success for optimising various systems and processes. In this paper, meaningful interpretation of super-additive and sub-additive inequalities has been used for maximising the stored elastic strain energy at a specified total displacement and for optimising the profit from an investment. 
Finally, meaningful interpretation of an algebraic inequality has been used for reducing uncertainty and the risk of incorrect prediction related to the magnitude ranking of sequential random events. The various examples from distinct application domains led to the formulation of the principle of non-contradiction: if the variables and the different parts of an abstract inequality can be interpreted as parts of a physical process, in the real world, the physical process must take course such that it stays consistent with the predictions from the abstract inequality.

The proposed method for improving reliability and reducing risk based on inequalities is domain-independent. It transcends mechanical engineering and can be applied as a tool for reliability improvement and risk reduction in many unrelated domains.

\section{REFERENCES}

1. Collins J.A. Mechanical design of machine elements and machines. John Wiley \& Sons, Inc.: New York, 2003.

2. Norton R.L. Machine design, an integrated approach, 3rd ed. Pearson International edition. 2006.

3. Pahl G., Beitz W., Feldhusen J. and Grote K.H. Engineering design. Springer: Berlin, 2007.

4. Childs P.R.N. Mechanical design engineering handbook. Elsevier: Amsterdam, 2014.

5. Budynas R.G., Nisbett J.K. Shigley's Mechanical engineering design, 10th ed. McGrawHill Education, 2015.

6. Mott R.L., Vavrek E.M., Wang J. Machine Elements in Mechanical Design, 6th ed. Pearson Education Inc., 2018.

7. Gullo L.G., Dixon J. Design for safety. Wiley: Chichester, 2018.

8. Todinov, M. Methods for reliability improvement and risk reduction. Wiley, 2019.

9. Kazarinoff N.D. Analytic Inequalities. Dover Publications, Inc: New York, 1961.

10. Engel A., Problem-solving strategies. Springer: New York, 1998.

11. Hardy G., Littlewood J.E., Pólya G. Inequalities. Cambridge Mathematical Library, Cambridge University Press, 1999.

12. Steele J.M. The Cauchy-Schwarz master class: An introduction to the art of mathematical inequalities. Cambridge University Press: New York, 2004.

13. Pachpatte B.G. Mathematical inequalities. North Holland Mathematical Library, vol.67, Elsevier: Amsterdam, 2005.

14. Sedrakyan H., Sedrakyan N. (2010). Algebraic Inequalities, Cham: Springer.

15. Ebeling C.E. Reliability and maintainability engineering. McGraw-Hill: Boston, 1997.

16. M. Xie and C. D. Lai, (1998) On reliability bounds via conditional inequalities, Journal of Applied Probability, 35(1), 104-114

17. Makri F.S., Psillakis Z.M. (1996), Bounds for reliability of k-within two-dimensional consecutive-r-out-of-n failure systems, Microelectronics Reliability, 36(3), pp.341-345.

18. Hill S.D., Spall J.C., Maranzano C.J. (2013)., Inequality-Based Reliability Estimates for Complex Systems, Naval Research Logistics, pp.367-374.

19. DeGroot M.H. 1986, Probability and statistics, 2nd ed. Reading: Addisson-Wesley Publishing Company.

20. Berg V.D and Kesten H. (1985). Inequalities with Applications to Percolation and Reliability, Journal of Applied Probability, 22 (3), pp. 556-569.

21. Kundu C., Ghosh A., (2017) Inequalities involving expectations of selected functions in reliability theory to characterize distributions, Communications in Statistics - theory and methods, 46(17) https://doi.org/10.1080/03610926.2016.1183784. 
22. Dohmen K., (2006). Improved Inclusion-exclusion identities and Bonferoni inequalities with reliability applications, SIAM J. Discrete Math., 16(1), 156-171. https://doi.org/10.1137/S0895480101392630.

23. Lewis, E.E. Introduction to Reliability Engineering. Wiley: New York, 1996.

24. O'Connor, P.D.T. Practical Reliability Engineering, 4e. Wiley: New York, 2002.

25. Dhillon B.S. Engineering systems reliability, safety, and maintenance. Boca Raton, New York: CRC Press, 2017.

26. Modarres M., Kaminskiy M.P., Krivtsov V. Reliability engineering and risk analysis, a practical guide, 3rd ed. CRC Press, 2017.

27. Rastegin A. Convexity inequalities for estimating generalized conditional entropies from below. Kybernetika, 2012; 48(2): 242-253.

28. Cloud M., Byron C., Lebedev L.P. Inequalities: with applications to engineering. Springer-Verlag: New York, 1998.

29. Thompson G. Improving maintainability and reliability through design. Professional Engineering Publishing Ltd.: London, 1999.

30. French M. Conceptual design for engineers. 3rd ed. Springer-Verlag Ltd: London, 1999.

31. Samuel A. and Weir J. Introduction to engineering design: Modelling, synthesis and problem solving strategies. Elsevier: London, 1999.

32. Todinov M.T., Reliability analysis of complex systems based on the losses from failures, International Journal of Reliability, Quality and Safety Engineering, 13 (2), 127-148, 2006.

33. Todinov, M.T., New models for optimal reduction of technical risks. Engineering Optimization 2013; 45 (6): 719-743.

34. Cover T. Pick the largest number, in Open Problems in Communication and Computation, edited by Cover T.M., and Gopinath B.New York: Springer-Verlag, 1987.

35. Alsina C. and Nelsen R.B. Charming Proofs: A Journey into Elegant Mathematics, Washington, DC: The Mathematical Association of America, 2010.

\section{APPENDIX. Algorithms in pseudo-code}

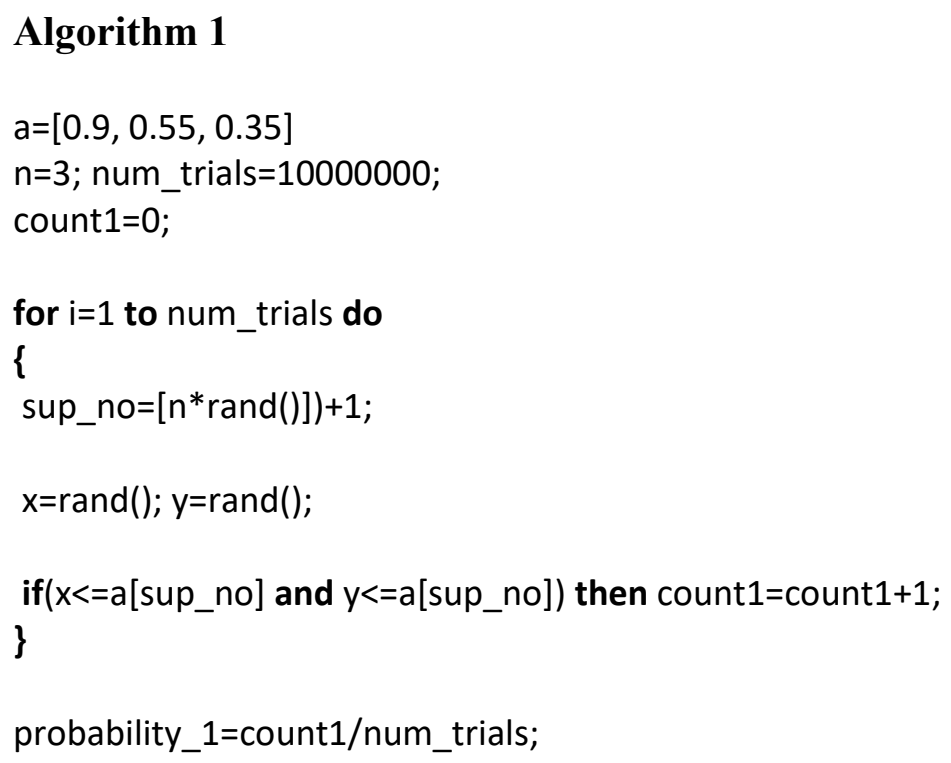

A supplier is selected randomly with the statement sup_no=[n* $\operatorname{rand}()])+1$, where $\left[\mathrm{n}^{*} \operatorname{rand}()\right]$ is the greatest integer part that does not exceed $n^{*}$ rand() and rand() is a function that returns a 
random number uniformly distributed in the interval $(0,1)$. The fractions of high-reliability components characterising the suppliers are specified in the array a[]. In the test case, $a[0.9$, $0.55,0.35]$ has been specified for the three suppliers. The test for two high-reliability components is performed by the conditional statement

$$
\text { if }(x<=a[\text { sup_no] and } y<=a[\text { sup_no] }) \text { then count1 =count } 1+1 \text {; }
$$

where $x$ and $y$ are two random numbers uniformly distributed in the interval $(0,1)$. If the compound condition

$$
\mathrm{x}<=\mathrm{a} \text { [sup_no] and } \mathrm{y}<=\mathrm{a} \text { [sup_no] }
$$

is true, this means that both components are high-reliability components in which case the counter count 1 is incremented. If the compound condition is false, the counter count 1 is not incremented. Consequently, the counter count1 tracks the number of instances where two high-reliability components have been selected. Finally, the probability of purchasing two high-reliability components from a randomly selected single supplier is obtained by dividing the content of the counter to the number of trials.

Simulating purchasing of two high-reliability components from two randomly selected suppliers is done in a similar fashion. The algorithm in pseudo-code is given next

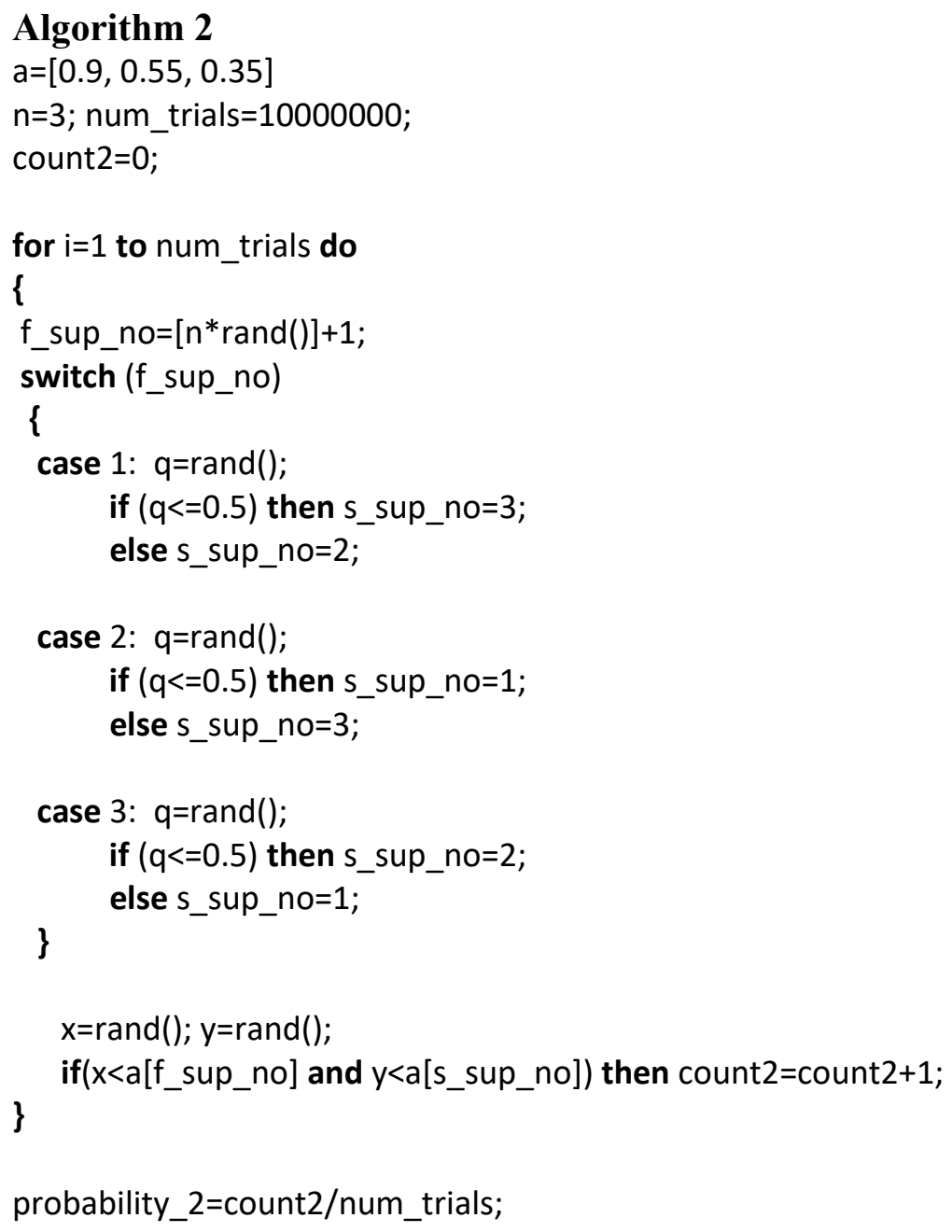

Initially, a first supplier is randomly selected, with the statement $f \_s u p \_n o=[n *$ rand()]+1;. Next, from the remaining two suppliers one more supplier is randomly selected by using the random variable $q$ to which the function rand() assigns a random value which is uniformly distributed over the interval $(0,1)$. Depending on whether the random value $q$ is smaller or greater than 0.5 , one of the remaining two suppliers is selected. The index of the randomly 
selected second supplier is kept in the variable s_sup_no. High-reliability components from the two suppliers are 'purchased' by generating two random numbers in the interval $(0,1)$ with the statements: $\mathrm{x}=\operatorname{rand}() ; \mathrm{y}=\operatorname{rand}() ;$. Next, if both $x$ and $y$ are smaller than the corresponding fractions for a high-reliability component, the purchased components are high-reliability components and the counter count 2

is incremented in the conditional statement

$$
\text { if }\left(x<\text { a[f_sup_no] and } y<a\left[s \_s u p \_n o\right]\right) \text { then count } 2=\text { count } 2+1 \text {; }
$$

Finally, the probability of purchasing two high-reliability components from two randomly selected suppliers is obtained by dividing the content of the counter count 2 by the number of simulation trials: probability_2=count2/num_trials;

The two simulation loops for purchasing components from a single random supplier and two random suppliers can be combined into a single simulation loop.

\section{Algorithm 3}

The algorithm is pseudo-code for confirming or disproving an inequality by a Monte simulation is given next.

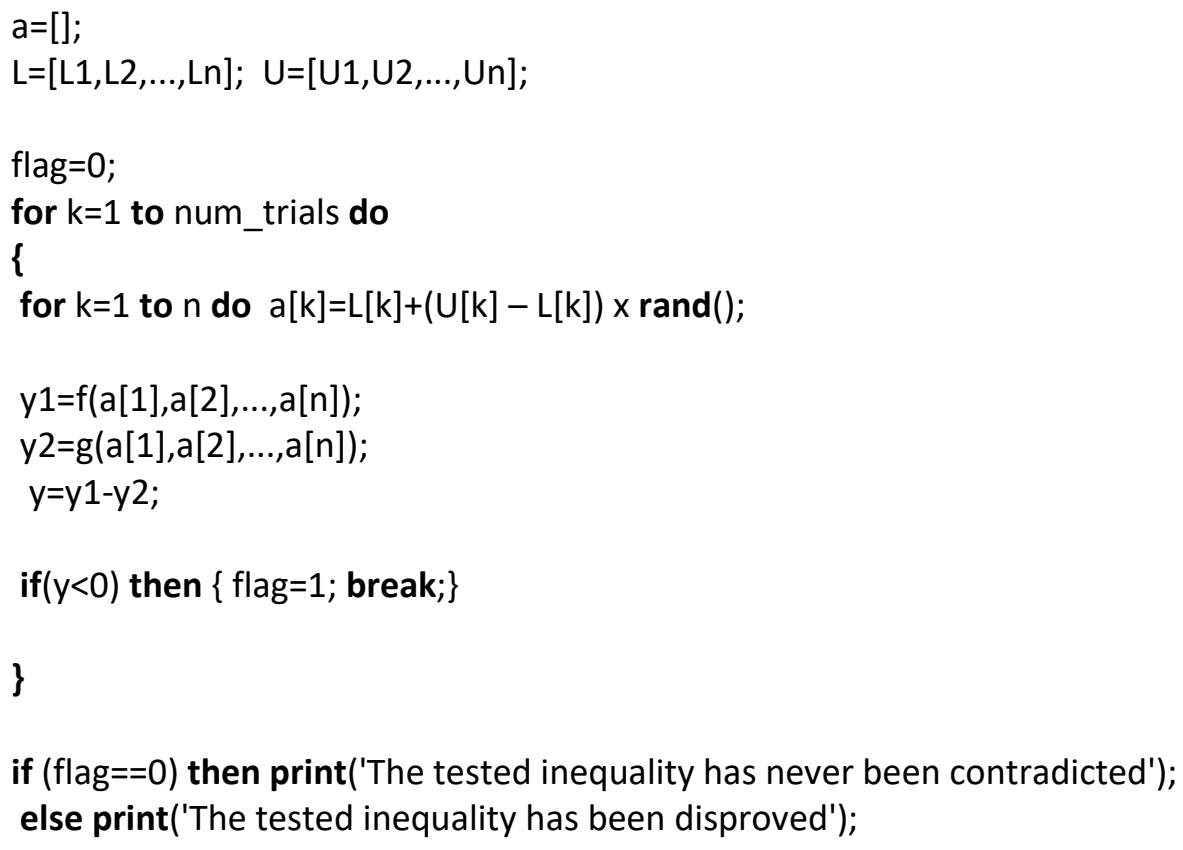

Initially, in the loop

for $k=1$ to $n$ do $a[k]=L[k]+(U[k]-L[k]) \times$ rand(),

random values are assigned to the variables a[k] entering the inequality.

This is done by calling the function rand(), which returns a random number uniformly distributed in the interval $(0,1)$. The value returned by rand() is transformed linearly by the statement $a[k]=L[k]+(U[k]-L[k]) \times$ rand() into a random value $a[k]$ uniformly distributed in the interval $(\mathrm{L}[\mathrm{k}], \mathrm{U}[\mathrm{k}])$.

Next, the left and right sides of the tested inequality are evaluated by calling the functions $f()$ and $g()$ with the statements

$$
y 1=f(a[1], a[2], \ldots, a[n]) ; y 2=g(a[1], a[2], \ldots, a[n]) ;
$$

The values returned by the functions are stored in the variables y1 and $y 2$. 
The difference $y=y 1-y 2$ is then checked for being smaller than zero. A difference smaller than zero corresponds to a case where the tested inequality has been contradicted. In the statement if $(y<0)$ then $\{$ flag=1; break; $\}$

if the tested inequality has been contradicted, a variable serving as flag is assigned value equal to one (flag=1) and the simulation loop is exited immediately with the statement 'break' because a counterexample has been found. At the end of the algorithm, the content of the variable flag is checked. If the flag remained zero during the simulations, this is an indication that the tested inequality has never been contradicted during the simulation trials which indicates that the tested inequality is probably true and a rigorous proof can be attempted. If, during the simulations, the variable flag has changed its value to one, this is an indication that there has been a combination of values for the variables entering the tested inequality for which the inequality does not hold. This means that the conjectured inequality has been falsified and there is no point in searching for a rigorous proof because a counterexample has been found which disproved the conjectured inequality.

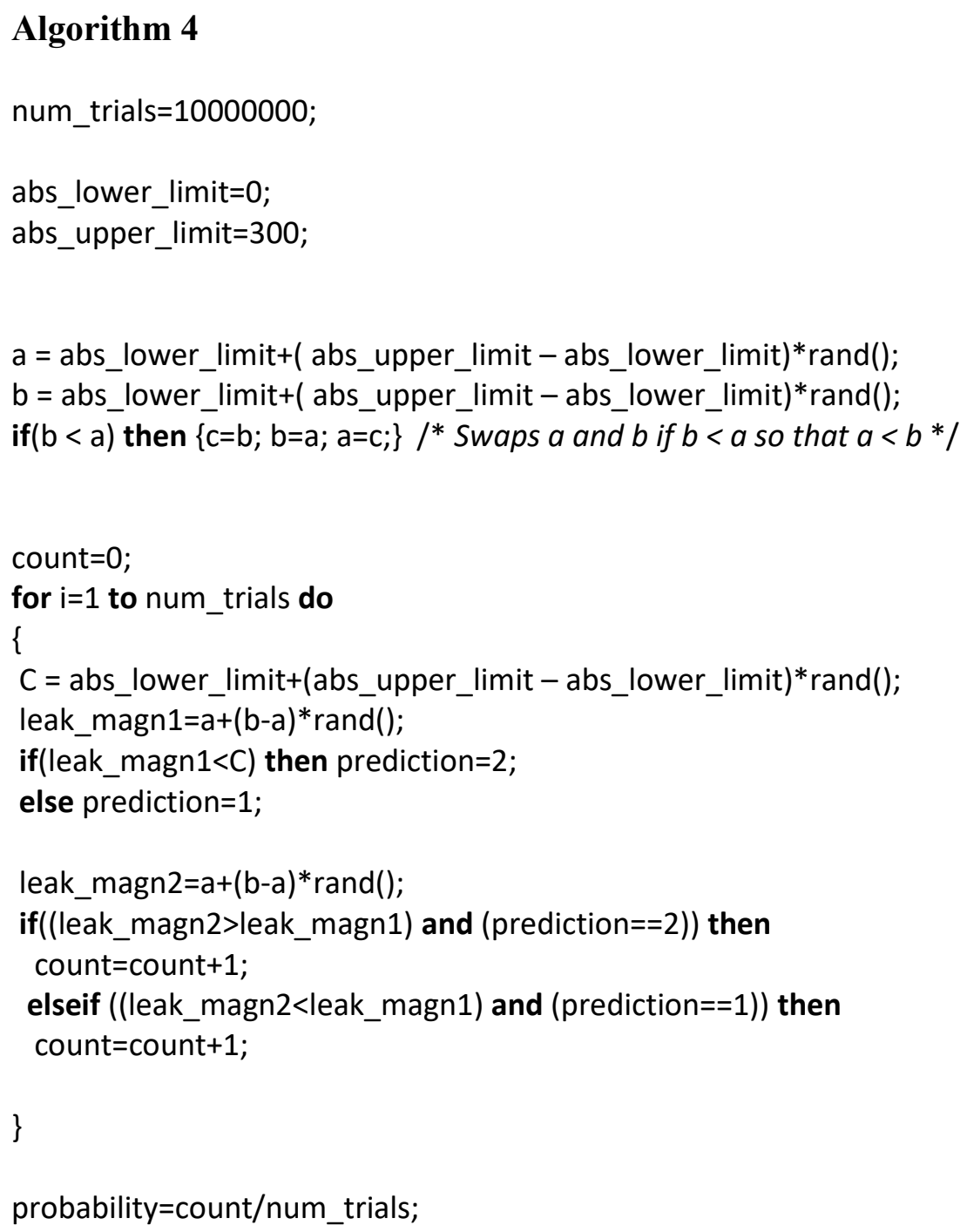

The function rand() returns a uniformly distributed random number in the interval $(0,1)$. The statements

$\mathrm{a}=$ abs_lower_limit+( abs_upper_limit - abs_lower_limit)*rand();

$b=$ abs_lower_limit+( abs_upper_limit - abs_lower_limit $) *$ rand(); 
generate a random interval with limits $a$ and $b$ for the leak magnitudes within the physically possible range $[0,300]$.

The statement

$$
C=\text { abs_lower_limit+(abs_upper_limit - abs_lower_limit)*rand(); }
$$

selects a random comparison threshold in the physically possible range $[0,300]$.

The statement leak_magn1=a+(b-a)*rand() generates a leak with random magnitude, uniformly distributed within the random interval with limits $a$ and $b$.

The variable 'prediction' predicts the ranking of the magnitude of the next leak with respect to the magnitude of the first leak. If prediction contains ' 1 ' the prediction is that the second leak will be smaller than the first leak; if prediction contains ' 2 ', the prediction is that the second leak will be bigger. The variable 'count' counts the number of trials during which the prediction is correct. This is done in the code fragment:

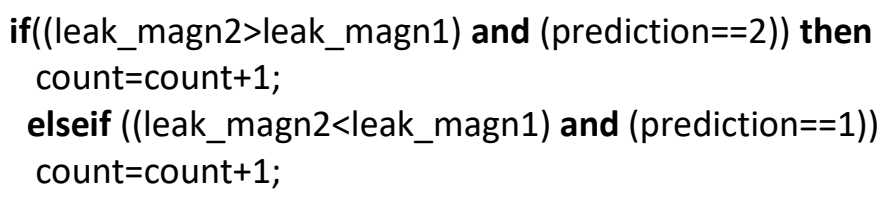

Michael Todinov has a background in mechanical engineering, applied mathematics and computer science. He received his $\mathrm{PhD}$ and his higher doctorate (DEng) from the University of Birmingham and is currently a professor in mechanical engineering in Oxford Brookes University, UK. Prof.Todinov pioneered research on reliability analysis based on the cost of failure, repairable flow networks and networks with disturbed flows, domain-independent methods for reliability improvement and risk reduction and reducing risk and uncertainty by using algebraic inequalities. In the area of reliability and risk, Prof. Todinov authored five books with reputable academic publishers and numerous research papers. In 2017, he received the prestige award of the Institution of Mechanical Engineers (UK) in the area of risk reduction in mechanical engineering. 